\title{
1 The Empirical Adaptive Wavelet Decomposition (EAWD): An adaptive decomposition for the variability analysis of observation time series in atmospheric science.

\author{
${ }^{1}$ Laboratoire de l'Atmosphère et des Cyclones, (LACy, UMR 8105 CNRS, Université de la Réunion, Météo- \\ France), Université de La Réunion, 97400 Saint-Denis de La Réunion, France \\ ${ }^{2}$ School of Chemistry and Physics, University of KwaZulu-Natal, Westville, Durban 4041, South Africa \\ ${ }^{3}$ Department of Physics and Astronomy, The University of New Mexico, Albuquerque, NM, USA \\ ${ }^{4}$ École Nationale Supérieure des Techniques Avancées, Paris, France \\ Correspondence to: olivier.delage@univ-reunion.fr
}

\begin{abstract}
Most observational data sequences in geophysics can be interpreted as resulting from the interaction of several physical processes at several time and space scales. As a consequence, measurements time series have often characteristics of non-linearity and non-stationarity and thereby exhibit strong fluctuations at different timescales. The variability analysis of a time series consists in decomposing it into several mode of variability, each mode representing the fluctuations of the original time series at a specific time-scale.

Such a decomposition enables to obtain a time-frequency representation of the original time series and turns out to be very useful to estimate the dimensionality of the underlying dynamics. Decomposition techniques very well suited to non-linear and non-stationary time series have recently been developed in the literature. Among the most widely used of these technics are the empirical mode decomposition (EMD) and the empirical wavelet transformation (EWT). The purpose of this paper is to present a new adaptive filtering method that combines the advantages of the EMD and EWT technics, while remaining close to the dynamics of the original signal made of atmospheric observations, which means reconstructing as close as possible to the original time series, while preserving its variability at different time scales.
\end{abstract}

KEY-WORDS: Variability Analysis, Complex Dynamics, Adaptive Filtering, Empirical Mode Decomposition (EMD), Empirical Wavelet Transformation (EWT), Non-Linear and Non-Stationary time series, Wavelet, Atmospheric observation.

\section{Introduction:}

Geophysical systems are mostly complex and the variability of the corresponding observation time series is characterized by large fluctuations at different time-scales. To analyze these fluctuations and the associated multiscale dynamics, some specific methods have been developed. The empirical mode decomposition (EMD) is a part of a more general signal processing method called Hilbert-Huang transform (HUANG et al., 1998) and consists in decomposing a signal in a self-adaptive way, into a sum of oscillating components named IMFs (Intrinsic Mode Function). Each IMF captures the repeating signal behavior at some particular time scale. Like the wavelet transform, the EMD technics reduces a time signal into a set of basis signals; unlike the wavelet transform, the basic functions are derived from the signal itself. The main advantages of the EMD method are to be fully adaptive, data driven and indeed close to the observed dynamics. As the EMD acts as a bank of bandpass filters (FLANDRIN et all 2004), the main limiting factor is the frequency resolution that may give rise to the mode mixing phenomenon where the spectral contents of some IMFs overlap each other (GAO et al., 2008). Although several techniques exist to overcome this problem (FOSSO et al., 2017, DELAGE et al., 2019), (Gilles, 2013) proposed an alternative one entitled "Empirical Wavelet Transform" (EWT), which consists in building a bank of filters from the segmentation of the original signal Fourier spectrum. This approach is similar to that used in the construction of both Littlewood-Paley and Meyers wavelets (MEYER, 1997). The heart of the EWT method lies on the segmentation of the Fourier spectrum based on the local maxima detection, in order to obtain a set of nonoverlapping segments. Because linked to the Fourier spectrum, the frequency resolution provided by the EWT is higher than that provided by the EMD and therefore allows to overcome the mode mixing problem (GILLES, 2013). Although the EWT technique enables to detect the relevant frequencies involved in the original time series fluctuations, such a technique does not allow to associate the detected frequencies to a specific mode of variability as EMD does. Because the EMD is closer to the observed dynamics than EWT, we developed in the present work a new approach called EAWD (Empirical Adaptive Wavelet Decomposition) based on the coupling of the EMD 
and EWT techniques. We use the spectral content of the IMFs obtained by the EMD to optimize the segmentation of the original time series Fourier spectrum required by EWT. This document is structured around four sections. The first section is devoted to the EMD and EWT techniques; in the second one, the new adaptative decomposition EAWD is described and explain why such a method can be considered as an optimization of the EMD. In the third section, the observation time series which are analyzed by the EMD and EAWD techniques are presented. Finally, in the last paragraph, the comparison of the results obtained respectively with the EMD and EAWD techniques are discussed.

\section{2-The existing approaches.}

\subsection{The Empirical Mode Decomposition (EMD).}

In 1998, HUANG et al. proposed an original method called the Empirical Mode Decomposition (EMD) that decomposes adaptively any signal into oscillatory contributions. In a nutshell, the EMD can be summarized as an iterative method, where the signal is considered as the superimposition of high and low frequency oscillations. At each iteration, high frequency oscillations components are separated from the low frequency oscillations, then reinjected as a new signal in the following iteration. The EMD is indeed directly controlled by the signal itself and not by some filtering operations as in the wavelet decomposition. More precisely, the decomposition is carried out at the scale of local oscillations with the low frequency modes are obtained as the mean value of an upper and a lower envelope computed as cubic splines interpolations between maxima and minima, respectively. By subtracting this component from the original signal, we obtain what is called an "Intrinsic Mode Function" (IMF). The procedure is then applied to the low frequency part as a new signal to decompose and successive oscillatory components are iteratively extracted from the original signal. The original time series $x(t)$ can be finally expressed as the sum of a finite number $\mathrm{N}$ of IMFs and a residual term $\mathrm{R}$, that cannot be assimilated to an oscillation.

$x(t)=\sum_{i=1}^{N} I M F_{i}(t)+R(t)$

The interesting fact about this algorithm is that it is highly adaptable and is able to extract the non-stationary part of the original signal. However, in practice the EMD technique presents some limiting factors. For example, some problems appear when some noise is present in the signal. To deal with this problem, (WU and WANG, 2009) introduced an EMD optimization entitled Ensemble Empirical Mode Decomposition (EEMD). The EEMD consists in computing several EMD decompositions of the original signal corrupted by different artificial noises. Then the final EEMD is the average of each EMD. This approach seems to stabilize the obtained decomposition

\subsection{Wavelet approaches.}

Wavelets are commonly used to analyze the variability of a signal. In the temporal domain, a wavelet basis is defined as the dilated with a parameter $\mathrm{s}>0$ and translated by $\mathrm{u} \in \mathrm{R}$ of a mother wavelet $\psi$ of zero-mean) as:

$\psi_{u, s}(t)=\frac{1}{\sqrt{s}} \psi\left(\frac{t-u}{s}\right)$.

For the wavelet decomposition of a time series $\mathrm{x}\left(\mathrm{t}_{\mathrm{i}}\right)$, the most used case is the dyadic case, $\mathrm{s}=2^{\mathrm{j}}$. Then the wavelet decomposition of $\mathrm{x}$ is obtained by computing the inner products $W_{x}(k, j)=\left\langle x, \psi_{k, j}\right\rangle$ where $\mathrm{j}$ represents the resolution level and $\psi_{k, j}(t)=\frac{1}{\sqrt{2^{j}}} \psi\left(\frac{t-k}{2^{j}}\right), k \in \mathbb{Z}$. The decomposition is then similar to a multiresolution analysis consisting in carrying out successive projections of $\mathrm{x}$ on a sequence of nested subspaces $V_{j} \in L^{2}(\mathbb{R}) j=[0, n]$, which leads to increasingly coarse approximations of $\mathrm{x}$ as $\mathrm{j}$ increases. The difference between two successive approximations resulting respectively from projections of $x$ on $V_{j-1}$ and $V_{j}$, contains the information of "details" which existed on the scale $2^{j-1}$ and which is lost on the scale $2^{\mathrm{j}}$. This information is contained in the subspace $\mathrm{W}_{\mathrm{j}}$ orthogonal to $V_{j}$ such that: $V_{j-1}=V_{j} \oplus W_{j}$, where $\oplus$ denotes the direct sum of vector subspaces. The orthogonal projection of $\mathrm{x}$ on $\mathrm{W}_{\mathrm{j}}$ gives the information of "details" at the resolution level $\mathrm{j}$. Wavelets $\left\{\psi_{k, j}(t), k \in \mathbb{Z}\right\}$ form a basis of $\mathrm{W}_{\mathrm{j}}$. According to the definition of a multiresolution analysis, there exists a function $\varphi(t)$ called a scaling function such that $\{\varphi(t-k), k \in \mathbb{Z}\}$ form a basis of $\mathrm{V}_{0}$ corresponding to the coarsest approximation of $\mathrm{x}$. The reconstruction of $\mathrm{x}$ is obtained by:

$x(t)=\langle x(t), \varphi(t)\rangle \cdot \varphi(t)+\sum_{j=1}^{N}\left\langle x(t), \psi_{k, j}(t)\right\rangle \cdot \psi_{k, j}(t)$ 
Where \langle\rangle represents the inner products. The approximation coefficients corresponding to the coarsest resolution level are given by $\langle x, \varphi\rangle$ and the details coefficients corresponding to the successive decreasing resolution level $\Delta s_{j}=\Delta s_{j-1} / 2$ are given by $\left\langle x, \psi_{k, j}\right\rangle$ as following:

$\left.\langle x(t), \varphi(t)\rangle=\int x(\tau) \overline{\varphi(\tau-t)} d \tau ;\left\langle x(t), \psi_{k, j}(t)\right\rangle=\int x(\tau) \overline{\psi_{k, j}(\tau-t}\right) d \tau$

\subsection{Empirical wavelets- The Empirical Wavelet Transformation (EWT).}

The essence of EMD is that the functions into which a signal is decomposed are all in the time domain and of the same length as the original signal, allowing time varying frequencies to be preserved. In this context Rilling et al. (2004) described the EMD to behave as a dyadic filter bank as those involved in multiresolution analysis. This can be interpreted as having several filters of overlapping frequency content that may give rise to the mode mixing phenomenon, which is defined as a single IMF either consisting of widely disparate scales, or similar scale residing in different IMF. In that case, spectral content of some IMFs overlaps each other. To overcome this problem Gilles (2013) proposed an alternative entitled "Empirical Wavelet Transform" (EWT). As the EMD technique acts as a filter bank in the spectral domain, the method proposed by Gilles (2013) consists in designing an appropriate wavelet filter bank from the segmentation of the original signal Fourier spectrum. The Fourier support $[0, \pi]$ is segmented into $N$ contiguous segments denoted $\Delta_{n}=\left[\omega_{n-1}, \omega_{n}\right]$.

The filter bank (MEYER, 1997; JAFFARD et al., 2001) is defined by the empirical scaling function and the empirical wavelets on each $\Delta_{\mathrm{n}}$ through the equations 5 and 6 , respectively:

$$
\widehat{\phi}_{n}(\omega)=\left\{\begin{array}{cc}
1 & \text { if }|\omega| \leq(1-\gamma) \omega_{n} \\
\cos \left[\frac{\pi}{2} \beta\left(\frac{1}{2 \gamma_{n}}\left(|\omega|-(1-\gamma) \omega_{n}\right)\right)\right] & \begin{array}{c}
\text { if }(1-\gamma) \omega_{n} \leq|\omega| \leq(1+\gamma) \omega_{n} \\
0
\end{array} \\
\text { otherwise }
\end{array}\right.
$$

$\widehat{\psi_{n}}(\omega)=\left\{\begin{array}{cc}1 & \text { if }(1+\gamma) \omega_{n} \leq|\omega| \leq(1-\gamma) \omega_{n+1} \\ \cos \left[\frac{\pi}{2} \beta\left(\frac{1}{2 \gamma \omega_{n+1}}\left(|\omega|-(1-\gamma) \omega_{n+1}\right)\right)\right] & \text { if }(1-\gamma) \omega_{n+1} \leq|\omega| \leq(1+\gamma) \omega_{n+1} \\ \sin \left[\frac{\pi}{2} \beta\left(\frac{1}{2 \gamma \omega_{n}}\left(|\omega|-(1-\gamma) \omega_{n}\right)\right)\right] & \text { if }(1-\gamma) \omega_{n} \leq|\omega| \leq(1+\gamma) \omega_{n} \\ 0 \text { otherwise }\end{array}\right.$

The function $\beta(\mathrm{x})$ is an arbitrary $\mathrm{C}^{\mathrm{k}}([0,1])$ function defined as:

$$
\beta(x)=\left\{\begin{array}{c}
0 \text { if } x \leq 0 \\
\beta(x)+\beta(1-x)=1 \\
1 \text { if } x \geq 1
\end{array} \forall x \in[0,1]\right.
$$

Many functions satisfy this property and the most used in the literature (DAUBECHIES, 1992) is:

$\beta(x)=x^{4}\left(35-84 x+70 x^{2}-20 x^{3}\right)$

The parameter $\gamma$ is chosen in respect to the following criterion:

$\gamma<\operatorname{Min}_{n}\left(\frac{\omega_{n+1}-\omega_{n}}{\omega_{n+1}+w_{n}}\right)$

The details and approximation coefficients are calculated by using Eqs 5 and 6 and are respectively given by inner products with the empirical wavelets $\psi_{n}$ and the scaling function $\phi_{1}$.

$W_{x}(n, t)=\left\langle x(t), \psi_{n}\right\rangle=\operatorname{IFFT}\left(X(\omega) \cdot \overline{\bar{\psi}_{n}(\omega)}\right)$

$W_{x}(1, t)=\left\langle x(t), \phi_{1}\right\rangle=\operatorname{IFFT}\left(X(\omega) . \overline{\varphi_{1}(\omega)}\right)$

Where $\mathrm{X}$ is the Fourier transform of the original signal $\mathrm{x},{ }^{-}$represents the complex conjugate, IFFT represents the inverse Fourier transform, $\psi_{n}$ and $\phi_{1}$ are respectively the results of the inverse Fourier transform of $\widehat{\psi_{n}}$ and $\widehat{\phi_{1}}$. 
The heart of the empirical wavelet transform lies in the segmentation of the original signal Fourier spectrum. In order to obtain a set of non-overlapping segments, the Fourier spectrum local maxima are detected. Each segment is centered around a group of local maxima composed of one or more local maxima. The limit between two contiguous segments, each of them characterized by a group of local maxima, is determined as the local minimum closest to the middle between the two local maxima groups. Many of the detected local maxima are irrelevant as their contributions to the original time series variability are negligible. The selection of the relevant local maxima requires setting a threshold, which is not always possible.

\section{The Empirical Adaptive Wavelet Decomposition (EAWD).}

The EMD enables to decompose the fluctuations of an observation data sequence into different time scales, and therefore allows to identify the modes of variability (IMFs) of the observed dynamics, as well as the frequency spectrum associated with each IMF. The cycle of each IMF provided by the EMD technique is identified as being the dominant frequency of its frequency spectrum.

One of the main advantages of the Empirical Wavelet Transform (EWT) is to overcome the mode-mixing drawback caused by the EMD technic. Indeed, EWT enables to extract the different variability modes of the signal by designing an appropriate wavelet filter bank in the Fourier domain of the original signal so that their supports do not overlap, which is equivalent to building a set of band-pass filters. The adaptability of the method lies on the fact that the filter supports must be constructed according to where the information is located in the Fourier spectrum of the original signal. The efficiency of the EWT method enables to represent the observed dynamics and depends strongly on the segmentation of the Fourier spectrum.

Although the EMD technique suffers from a lack of theory, it enables to extract several modes of variability (IMFs), the whole of which is close to the physics underlying a time series and reflects the observed dynamics. However, the spectral content supports of the IMFs returned by the EMD overlap and give rise to the phenomenon of mode mixing. The EWT technique enables to overcome the problem of mode mixing by being based on a segmentation of the Fourier spectrum of the original signal in order to then extract from the segment modes of variability in the time domain. However, the EWT method is unable to associate the detected frequencies with a time scale like the EMD method does. The main idea of the proposed EAWD method is to combine the advantages of the EMD and EWT techniques by setting non-overlapping groups of local maxima from the spectral contents of the IMFs returned by the EMD technic. Each group of local maxima will be associated to a support of the original signal Fourier spectrum segmentation. The boundaries of each of these supports will be set as the local minima located between two consecutive groups of local maxima. As the EMD acts like a bank of dyadic band-pass filters: the result of each of these filters, in the frequency domain, being composed of a set of local maxima relative to a specific timescale in which the resolution is divided by two in comparison with the timescale immediately higher. Considering that a timescale is characterized by the set of values in the range of $\left[2^{n}, 2^{n+1}\right]$, to carry out a segmentation of the Fourier spectrum of the original time series, it is necessary to determine to which timescales the local maxima group of an IMF belongs to.

The proposed Fourier spectrum segmentation algorithm is based on two main points:

1- Two consecutive IMFs contain at most two different dominant frequencies.

2- The list of local maxima contained in the spectrums of two consecutive IMFs are subdivided into three groups: the local maxima belonging to IMFi: ML1- the local maxima belonging to the intersection of $\mathrm{IMF}_{\mathrm{i}}$ and $\mathrm{IMF}_{\mathrm{i}+1}$ : MLINTER- the local maxima belonging to $\mathrm{IMF}_{\mathrm{i}+1}$ : ML2.

The proposed EAWD algorithm is composed of three steps described in the diagrams below (see Figure 1, Figure 2, Figure 3): 


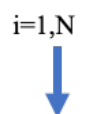

Calculate DSS $\mathrm{i}_{\mathrm{i}}$ the spectral density of $\mathrm{IMF}_{\mathrm{i}}$ as a function of the frequency : $f \rightarrow D S S_{i}[f]$

Identify $\mathrm{fd}_{\mathrm{i}}$ the dominant frequency of $\mathrm{IMF}_{\mathrm{i}}$.

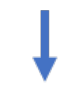

Determine among the local maxima $\mathrm{ml}_{\mathrm{j}}$ de $\mathrm{DSS}_{\mathrm{i}}$ the list $\mathrm{Ml}_{\mathrm{i}}$ of those whose energy contribution is greater than $1 \%$

$$
M L_{i}=\left\{m l_{j} \text { so that } \frac{m l_{j}}{\sum_{j} m l_{j}}>1 \%\right\}
$$

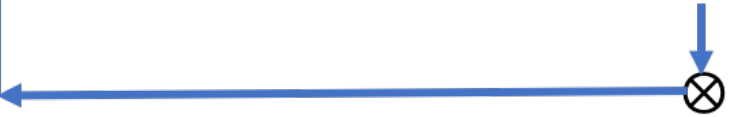

Figure 1: Estimation of the spectral density for each IMF and selection of significant local maxima.

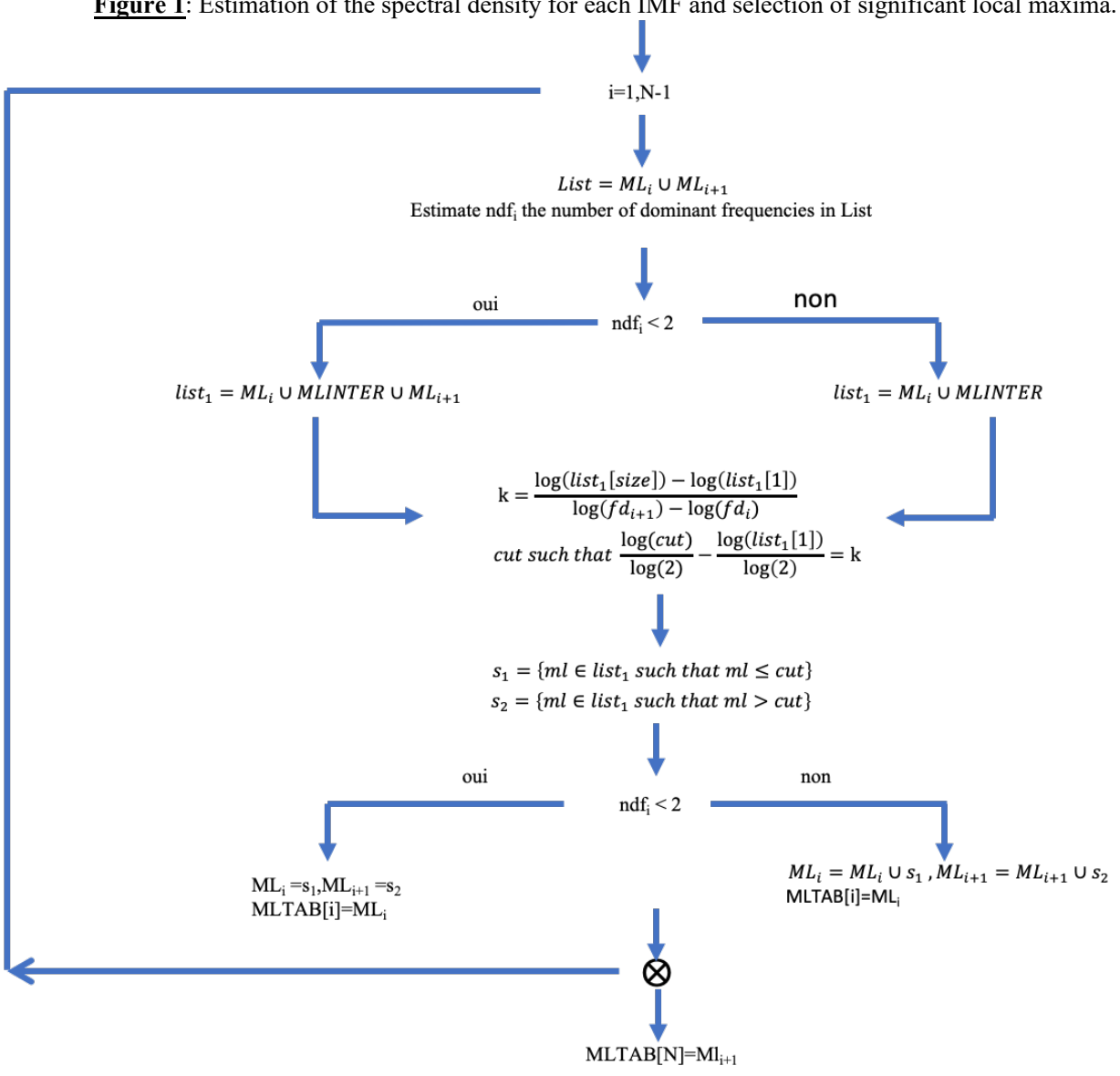

Figure 2: Calculation of the matrix MLTAB. Each row of MLTAB contains the list of significant local maxima present in the spectrum of an IMF. The rows of MLTAB have no common elements and therefore MLTAB can be seen as a set of non-overlapping local maxima groups and represents a segmentation of the original signal Fourier spectrum. 
1

"size" represents the length of list1 and cut is the boundary between two consecutive local maxima groups $\mathrm{ML}_{\mathrm{i}}$ and $\mathrm{ML}_{\mathrm{i}+1}$. The parameter $\mathrm{k}$ can be interpreted as the ratio between the number of scales contained in list 1 and the number of scales in the range $\left[\mathrm{fd}_{\mathrm{i}}, \mathrm{fd}_{\mathrm{i}+1}\right]$ of two consecutives dominant frequencies.

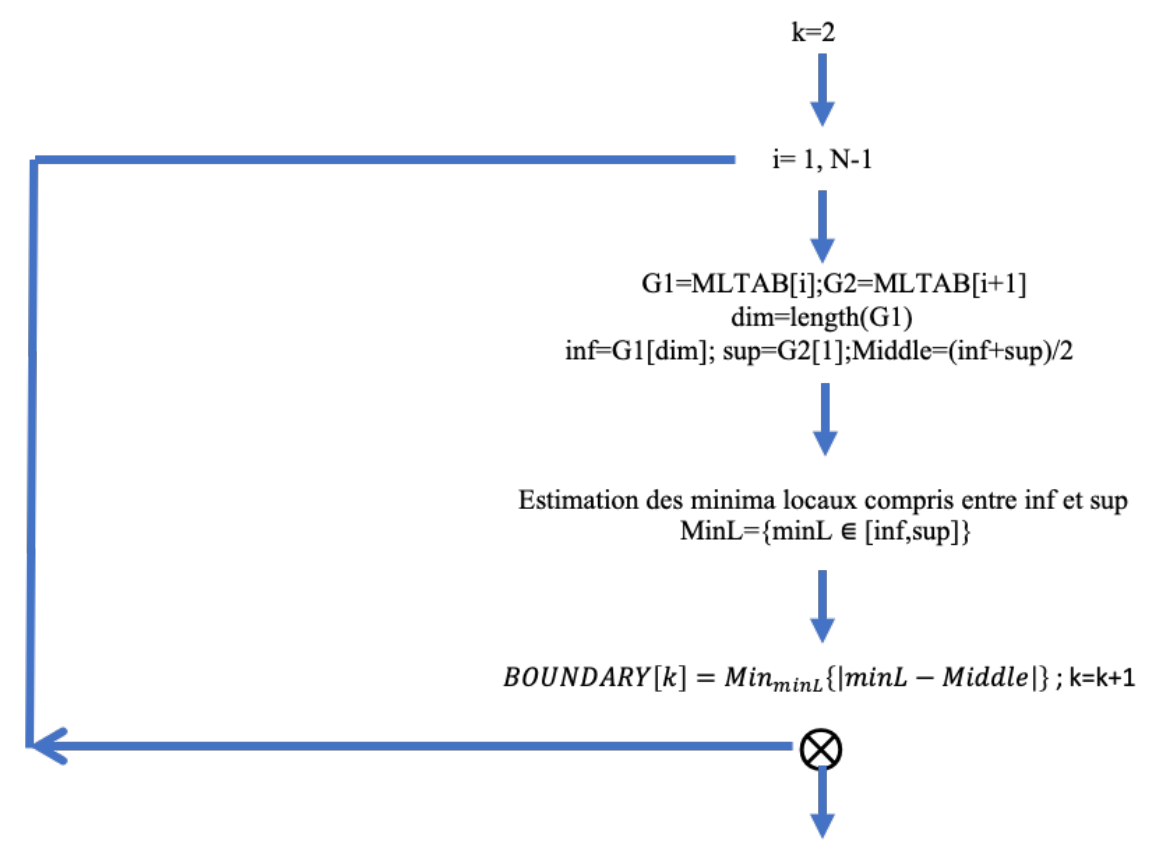

$\mathrm{G}=$ MLTAB[1]; dim=length[G];inf $=\mathrm{G}[1] ;$ sup $=\mathrm{G}[\mathrm{dim}] ;$ Middle $=($ inf + sup $) / 2$ $\operatorname{MinL}=\{\operatorname{minL} \in[$ inf,sup] $\} ;$ var=Middle-(BOUNDARY[2]-Middle)

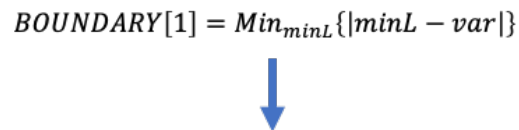

$\mathrm{G}=\mathrm{MLTAB}[\mathrm{N}] ; \mathrm{dim}=$ length[G];inf $=\mathrm{G}[1] ; \sup =\mathrm{G}[\mathrm{dim}] ;$ Middle $=($ inf + sup $) / 2$ $\operatorname{MinL}=\{\operatorname{minL} \in[$ inf,sup $]\} ;$ var=Middle $+($ Middle-BOUNDARY $[N-1])$

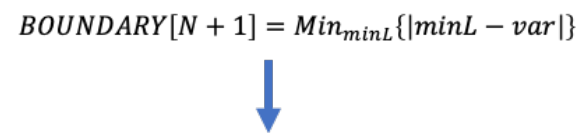

Run EWT technic from the BOUNDARY vector

Figure 3: Calculation of the BOUNDARY vector representing the boundaries between the local maxima groups contained in the matrix MLTAB. The EWT technique can be run from BOUNDARY.

\section{Time series analysis and results.}

EMD and EAWD technics presented above have been applied to an experimental time series of observations resulting of 41-years monthly Total Columns of Ozone (TCO), in Dobson units, recorded from 1979 to 2019 on Réunion Island $\left(21^{\circ} \mathrm{S}\right.$, $\left.55.5^{\circ} \mathrm{E}\right)$.

This TCO time series has been elaborated by merging satellite data (OMI and TOMS) and ground-based observations recorded by a SAOZ (Zenithal Observation Analysis System) spectrometer (Pommereau and Goutail, 1988) installed at 
Saint Denis, Réunion Island in 1993. The method used for merging satellite and ground data to obtain an homogeneous series is explained in Pastel et al., 2014.

Despite its low abundance, ozone plays an important role in the Earth's atmosphere. It is mainly produced in the tropical stratosphere and transported by the large-scale circulation called the Brewer-Dobson circulation to higher latitudes. In the stratosphere, ozone acts like a filter and prevents incident solar ultraviolet (UV) radiations from reaching the ground, thus protecting the biosphere. The significant depletion in the ozone layer since the late 1970s has revealed the importance of ozone in the climate system and the associated environmental and health risks. The TCO is a parameter that measures the abundance of ozone over a given location. It is given in Dobson Unit (DU) and consists of $\sim 90 \%$ stratospheric ozone and $\sim 10 \%$ tropospheric ozone.

The monthly means TCO time-series obtained over Reunion site is depicted on Figure 4.

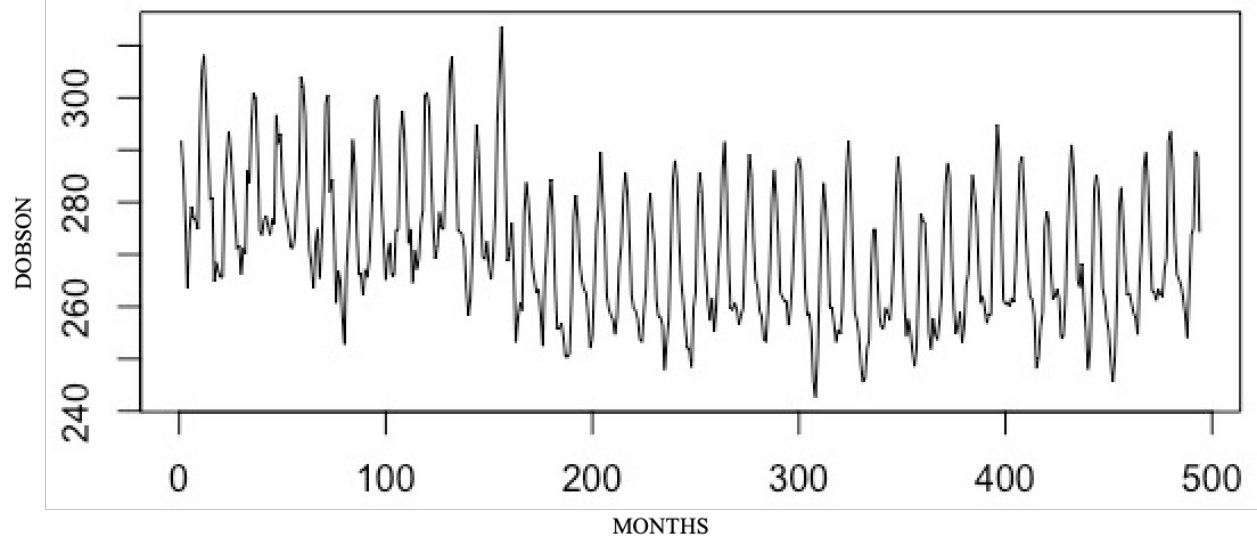

Figure 4: Monthly time-series of total ozone columns in Dobson over Reunion Island from 1979 to 2019. Time axis is expressed in months.

The EEMD technique was applied first to Reunion ozone time series presented in Figure 4. Considering the size of the original time series, 7 IMFs were found in addition to the residual mode. Generally, among IMFs returned by the EEMD technic, some of them present poor correlations with the original signal and therefore have a weak contribution in the variability of the original series. Such IMFs are qualified as irrelevant. Relevant IMFs are discriminated from the irrelevant ones by using two criteria. The first of these criteria uses the Pearson's correlation to estimate the degree of correlation of each IMF with the original signal by setting a threshold. A commonly threshold used in the literature (Ayenu-Prah et al., 2010) is expressed as:

$$
\tau=\frac{\max \left(\operatorname{Cor}\left(I M F_{i}, x\right)\right)}{\left(10 * \max \left(\operatorname{Cor}\left(I M F_{i}, x\right)\right)\right)-3}
$$

In the equation $(12), \operatorname{Cor}\left(\mathrm{IMF}_{\mathrm{i}}, \mathrm{x}\right)$ stands for the Pearson's correlation coefficient between the $\mathrm{i}^{\text {th }} \mathrm{IMF}$ and the original signal $\mathrm{x}(\mathrm{t})$, i.e.:

$\operatorname{Cor}\left(I M F_{i}, x\right)=\frac{\operatorname{Cov}\left(I M F_{i}, x\right)}{\operatorname{sd}\left(I M F_{i}\right) * s d(x)}$

Where sd represents the standard deviation and $\operatorname{cov}(.,$.$) the covariance.$

The second criterion determines the energy contribution of each IMF compared to the energy contained in the original signal. The energy contribution of each IMF is calculated as a percentage as follows:

$\operatorname{CENR}=\left(\frac{E_{i}}{E_{x}}\right) * 100=\frac{\operatorname{var}\left(I M F_{i}\right)}{\operatorname{var}(x-\bar{x})} * 100$

Where $E_{i}=\sum_{1}^{N} I M F_{i}^{2}(t)=\operatorname{var}\left(I M F_{i}\right)$ because each IMF is a zero-mean function by construction. $E_{x}=\sum_{1}^{N} x^{2}(t)=$ $\operatorname{var}(x-\bar{x})$.

The threshold set for this second criterion being $1 \%$.

IMFs whose degree of correlation is less than $\tau$ and which energy contribution is less than $1 \%$ are said to be irrelevant. The irrelevant IMFs are added to the residual mode R returned by the EEMD to form the trend of the original signal. Such IMFs to be part of the trend of the observed dynamics must not be too oscillating and are therefore contiguous to the residue. 
Once the relevant IMFs identified and selected, the results obtained from the Reunion TCO time-series are displayed on figure 5 below. Taking into account the selection procedure above, among the 7 IMFs initially identified, the first 5 are relevant and IMFs 6 and 7 are added to the residual mode.

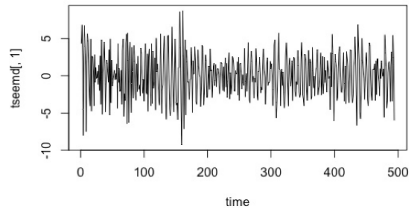

IMF1-CYCLE:6 months

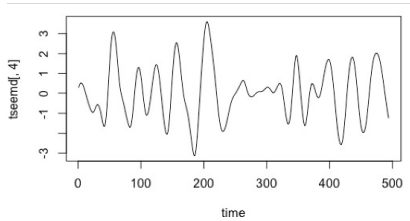

IMF4-CYCLE: 3.8 year

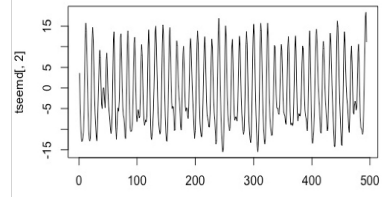

time

IMF2-CYCLE: 1 year

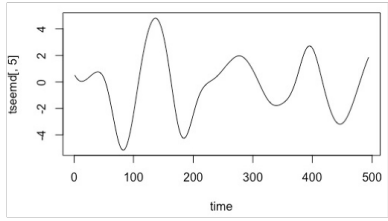

IMF5-CYCLE: 10.4 years

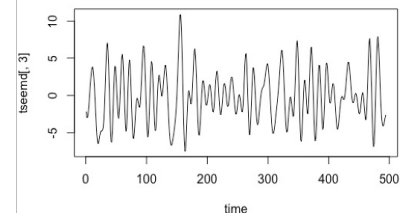

MF3-CYCLE: 1 year

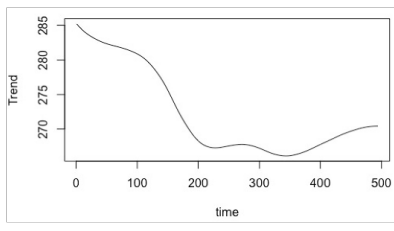

Figure 5: Relevant IMFs and Trend from the EEMD applied to Reunion total columns of ozone time-series, from 1979 to 2019.

For each IMF, the cycle defined as the period associated with the dominant frequency is specified. Mode mixing occurs in the IMFs 2 and 3 as they show the same period (1 year).

To estimate the accuracy of the residual returned by the EEMD, it is compared with the trend of the original signal obtained from a moving window which size has been set at the maximum of the relevant IMFs cycles i.e. 125 months (10.4 years).

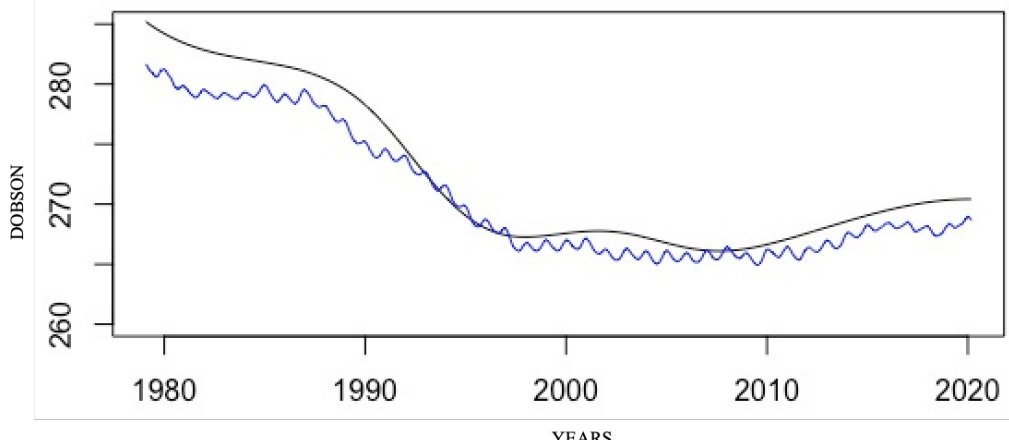

Figure 6: Trend obtained from the EEMD technique (black curve). The trend of the original signal obtained from a moving window (blue curve) with a 125 -months size (i.e. 10.4 years).

The accuracy of the residual R returned by the EEMD compared to the trend of the original signal by using a moving window Tmb was estimated using the following expression:

$P r_{E E M D}=\sqrt{\frac{\left(\sum_{i=1}^{N}(R(i)-T m b(i))^{2}\right) / N}{\overline{T m b}}} * 100=0.75 \%$

Where $\mathrm{N}$ is the original time-series length and ... represent the mean operator.

To overcome the mixing mode occurring in the IMFs 2 and 3 returned by the EEMD technique, we have applied the EAWD technique to the Reunion TCO time-series. Results obtained with EAWD are displayed in figure 7 below: 


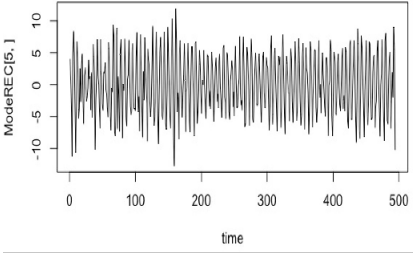

EAWD1-CYCLE: 6 months

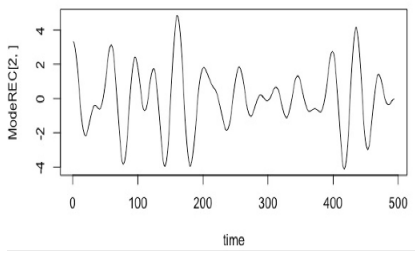

EAWD4-CYCLE: 3.8 years

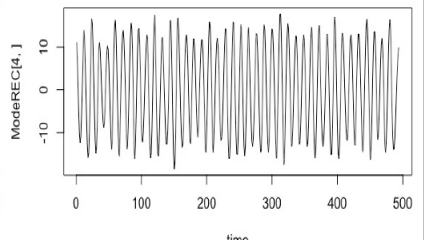

EAWD2-CYCLE: 1 year

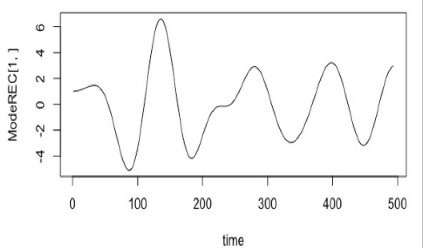

EAWD2-CYCLE: 10.4 years.

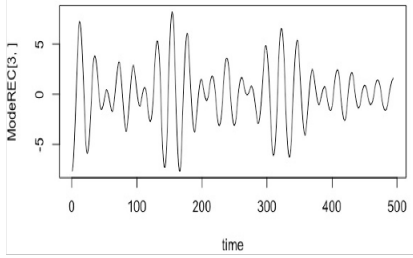

EAWD3-CYCLE: 1.7 year

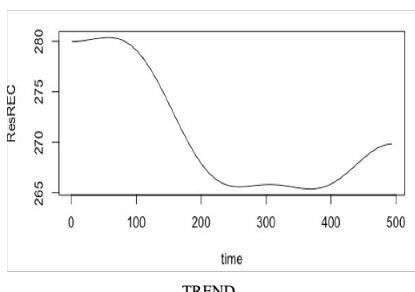

Figure 7: Reunion total ozone time-series EAWD decomposition results.

Similarly, the accuracy of the residual returned by the EAWD technic has been estimated and compared to that returned by the EEMD.

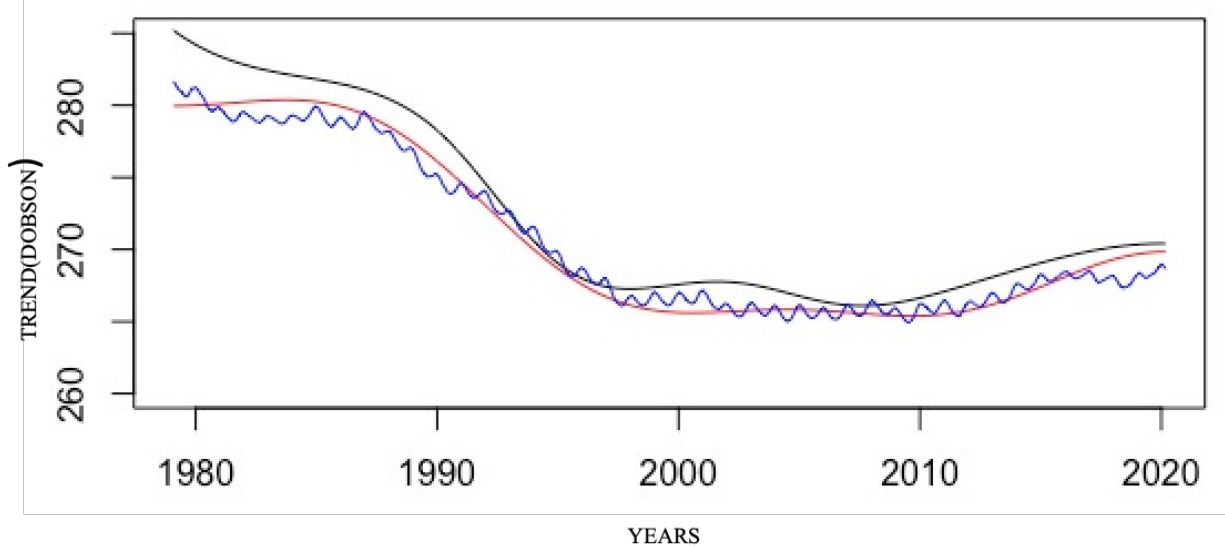

Figure 8: The residual Trend $_{E A W D}$ returned by the EAWD technique (red curve). The trend of the original signal obtained from a moving window (blue curve) whose size has been fixed at 125 (i.e. 10.4 years). The residual returned by the EEMD technic (black curve).

Likewise, the accuracy of the residual returned by the EAWD technique has been estimated using equation (12):

$\operatorname{Pr}_{\text {EAWD }}=0.3 \%$.

The EAWD trend expressed in DU/decade is shown in the figure below:

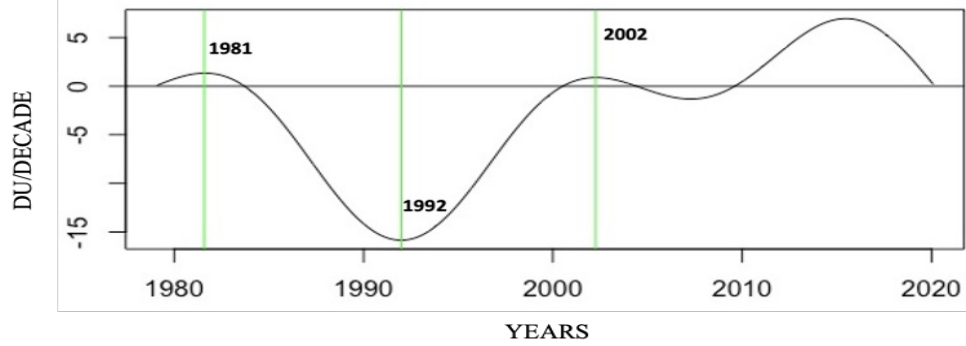

Figure 9: The DU/decade residual returned by the EAWD technique 
1

The accuracy of the DU/decade EAWD residual in Fig.9 is calculated as $2 * \operatorname{PrEAWD}_{\mathrm{E}}$, that is $0.6 \%$.

\section{Consistency of the EAWD method.}

The method commonly employed in atmospheric physics to determine variability and trend of observation timeseries is to use a multilinear regression model. This type of approach has been used very often (Randel and Thompson 2011; Nair et al., 2013; Bourassa et al., 2014; Gebhardt et al., 2014; Eckert et al., 2014 or Toihir et al, 2018).

The main point of this method lies in the a priori knowledge of the atmospheric climate forcing's that control the variability of the time series studied. The ozone-QBO relationship has been discussed in several papers as well as ENSO influence on ozone variability (Butchart et al. (2003), Brunner et al. (2006), Randel and Thompson, (2011)). Other papers have shown the role of solar flux in the temporal variability of ozone (Randel and Wu, 2007).

To validate results obtained with the EAWD method, we used the multilinear regression model "Trend-Run" developed at the University of Reunion and already used in many studies (Bencherif et al., 2006; Bègue et al., 2010, Toihir, 2018).

Climate forcing's used as input to TREND-RUN model are QBO, ENSO and solar flux. A linear function is used to estimate the long-term linear trend of the series. The trend is then estimated by calculating the slope of the normalized linear function. As the variability of ozone is also affected by the annual and semi-annual oscillations (AO and SAO), these two analytic oscillations are also used as input to the model. The TREND-RUN model as well as the climate forcing's used as (ENSO, QBO, solar flux at $10.7 \mathrm{~cm}$ ) are described in detail in Toihir et al. 2018.

The results obtained here with TREND-RUN on the 1979-2019 time-series are consistent with those obtained by Toihir et al., 2018, on a shorter total ozone series at Réunion Island (1998-2013).

The trend obtained from TREND-RUN model over the whole monthly mean ozone series from 1979 to 2019 is $1.11 \pm 0.19 \% /$ decade. The error is estimated from the standard deviation of the residual restitute by the model. The variability components returned by the EAWD method are in agreement with the climatic forcing's used as input to TREND-RUN model and the energy contributions respectively estimated by the TREND-RUN model and the EAWD technic are reported in the table below

\begin{tabular}{|c|c|c|c|c|}
\hline IMF & CYCLE & Corresponding proxy used in TREND-RUN model & $\begin{array}{c}\text { Energy contribution (\%) } \\
\text { TREND-RUN }\end{array}$ & \begin{tabular}{c} 
Energy contribution (\%) EAWD \\
\hline 1
\end{tabular} \\
\hline 2 months & SAO & $11 \%$ & $12.2 \%$ \\
\hline 3 & 12 months & AO & $66 \%$ & $58.4 \%$ \\
\hline 4 & 45 months & QBO & $3.1 \%$ & $4.7 \%$ \\
\hline 5 & 125 months & ENSO & $0.2 \%$ & $1.8 \%$ \\
\hline
\end{tabular}

Table1: Comparison of total ozone variability in Réunion (from 1979 to 2019) obtained with TREND-RUN model and the EAWD method.

The main advantage of the EAWD technic for analyzing the ozone variability from an observational time series is to extract auto adaptively the modes of variability implicitly contained in the original time series without requiring any a priori knowledge of the atmospheric forcing's controlling the ozone variability. It can be seen that the results returned by the EAWD method are in complete agreement with those returned by the TREND-RUN model. On the other hand, the energy contributions of the various modes of variability obtained with the EAWD method are compatible with those obtained with the TREND-RUN method (Table 1).

The EAWD technic enables to visualize the evolution of the trend over the whole period studied contrary to the trend returned by the TREND-RUN model which is linear over the whole duration of the series. This gives us much more precise information about the long-term evolution of the series, results in Fig 9, show that ozone levels in Reunion Island decreases from 1981, stops decreasing in 1992 and starts rising from 2002.

\section{Conclusion.}

Even though, the EMD adaptability seems useful for many applications, the main issue with EMD lies on its lack of theory. To overcome this lack of rigor, the EWT technic proposes a new approach introducing a way of building adaptive wavelets and thus extracting the different oscillatory modes from a signal by designing an appropriate filter bank. The heart of the EWT technic lies in the segmentation of the Fourier spectrum of the original signal. 
On the other hand, the EEMD returned the fluctuations of the signal at several timescales corresponding with the physics implicitly contained in the observation time series. The main idea of the EAWD technic is to use the spectral information contained in the different timescales returned by the EEMD and to ensure that their supports don't overlap, to segment the Fourier spectrum of the original signal. The results obtained using the EAWD technic are in good agreement with those obtained with the multilinear regression model TREND-RUN and from the comparison between results obtained respectively with the EMD and EAWD technics, it emerges that the EAWD technic enables to overcome the mode-mixing problem while providing a trend with better accuracy than that obtained with the EMD technic. The EAWD results from a coupling between the EEMD and EWT technics combining the rigor of the EWT by using wavelets, while keeping the behavior of the signal in the timescales returned by the EEMD. In this context, the EAWD technique can be seen as an optimization of the EMD.

\section{References.}

1- Huang N. E., Z. Shen, S. R. Long, M. C. Wu, H. H. Shih, Q. Zheng, N.- C. Yen, C. C. Tung, and H. H. Liu, The empirical mode decomposition and the Hilbert spectrum for nonlinear and non-stationary time series analysis, Proc. of the Royal Society of London A: Math., Physical and Engineering Sciences, vol. 454, no. 1971, pp. 903995, 1998.

2- Flandrin P., G. Rilling, and P. Gonçalvès, "Empirical Mode Decomposition as a filter bank”, IEEE Sig. Proc. Soc. Lett, vol 11, no 2, pp 112-114, 2004

3- Gao Y., G. Ge, Z. Sheng, E. Sang, "Analysis and solution to the mode mixing phenomenon in EMD, IEEE Congress on image and signal processing, 2008.

4- Olav.B. Fosso, M.Molinas, "Method for mode mixing separation in Empirical Mode decomposition, arXiv:1709.05547v1 [stat.ME], 16 septembre 2017.

5- Delage O., T. Portafaix, H. Bencheriff, G. Guimbretière, R. T. Loua, »Multi-scale Variability Analysis of Time Series in Geophysics by using the Empirical Mode Decomposition”, Processing SAGA 2019.

6- Gilles J., "Empirical Wavelet Transform », IEEE Trans. On Signal Processing, vol XX, NO XX, February 2013.

7- Daubechies I., "ten Lectures on Wavelets", Society for Industrial and Applied Mathematics, Cbms-Nsf Regional Conferences Series in Applied Mathematics, 1992

8- Wu Z., N.E. Huang, Ensemble Empirical Mode Decomposition: A Noise-Assisted Data Analysis Method », Advances in Adaptive Data Analysis / Vol 01, No 01, PP 1-41, 2009.

9- Jaffard S., Y.Meyer, R.D Ryan, "Wavelets Tools for Science and Technology”, SIAM 2001.

10- Meyer Y., "Wavelet, Vibrations and Scaling",American Mathematical Society, Centre de Recherches Mathématiques, 1997.

11- Ayenu-Prah A.Y., N. Attoh-Okine, "A Criterion for Selecting Relevant Intrinsic Mode Functions in Empirical Mode Decomposition", Advances in Adaptive Data Analysis, 2010.

12- Begue N, Bencherif H, Sivakumar V, Kirgis G, Mze N and Leclair de Bellevue J., Temperature variability and trends in the UT-LS over a sub-tropical site, Reunion, Atmos. Chem. Phys., 10, 8563-8574, 2010

13- Bencherif H., R. Diab, T. Portafaix, B. Morel, P. Keckhut, and A. Moorgawa, Temperature climatology and trend estimates in the UTLS region as observed over a southern subtropical site, Durban, South Africa, Atmos. Chem. Phys., 6, 5121-5128, 2006

14- Toihir, A. M., Portafaix, T., Sivakumar, V., Bencherif, H., Pazmiño, A., and Bègue, N.: Variability and trend in ozone over the southern tropics and subtropics, Ann. Geophys., 36, 381-404, https://doi.org/10.5194/angeo-36-381-2018, 2018 